\title{
The Arrangement of Endosperm Cells and Development of Starch Granules Are Associated With the Occurrence of Grain Chalkiness in Japonica Varieties
}

\author{
Bo Peng ${ }^{1}$, Dong-Yan Kong ${ }^{1}$, Tondi-Yacouba Nassirou ${ }^{2}$, Yu Peng ${ }^{3}$, Lu-Lu He ${ }^{1}$, Yan-Fang Sun ${ }^{1}$, Rui-Hua Pang ${ }^{1}$, \\ Xiao-Hua Song ${ }^{4}$, Juan Peng ${ }^{5}$, Hui-Long $\mathrm{Li}^{4}$, Gui-Ying Guo ${ }^{4}$, Jin-Tiao Li ${ }^{1}$, Lin Liu ${ }^{3}$, Shi-Zhi Song ${ }^{4}$, Qi-Ying Zhou ${ }^{1}$, \\ Bin Duan ${ }^{4} \&$ Hong-Yu Yuan ${ }^{1}$ \\ ${ }^{1}$ College of Life Sciences and Institute for Conservation and Utilization of Agro-bioresources in Dabie \\ Mountains, Xinyang Normal University, Xinyang, China \\ ${ }^{2}$ Plate Forme Agroécologique Multi Acteurs Raya Karkara Du Niger, Niamey, Niger \\ ${ }^{3}$ School of Science and Technology, Xinyang University, Xinyang, China \\ ${ }^{4}$ Xinyang Academy of Agricultural Science, Xinyang, China \\ ${ }^{5}$ Xinyang Station of Plant Protection and Inspection, Xinyang, China \\ Correspondence: Bo Peng, College of Life Sciences and Institute for Conservation and Utilization of \\ Agro-bioresources in Dabie Mountains, Xinyang Normal University, Xinyang 464000, China. E-mail: \\ pengbo@xynu.edu.cn \\ Tondi-Yacouba Nassirou, Plate Forme Agroécologique Multi Acteurs Raya Karkara Du Niger, BP: 11271, \\ Niamey, Niger. E-mail: tnassirou2009@gmail.com \\ Hong-Yu Yuan, College of Life Sciences and Institute for Conservation and Utilization of Agro-bioresources in \\ Dabie Mountains, Xinyang Normal University, Xinyang 464000, China. E-mail: yhongyu92@163.com
}

Received: March 18, 2018

doi:10.5539/jas.v10n7p156

\author{
Accepted: April 30, $2018 \quad$ Online Published: June 15, 2018 \\ URL: https://doi.org/10.5539/jas.v10n7p156
}

\begin{abstract}
The endosperm chalkiness in grains is one of the most important traits in rice grain qualities, and grain chalkiness directly determines grain quality and market price. Therefore, the endosperm chalkiness reduction is an important goal of breeding scientist in rice. However, little is known about the relationship of the formation of rice endosperm chalkiness and the morphological structure and development in fragrant japonica and conventional japonica varieties. In this study, we have mainly investigated the chalkiness characters such as degree of endosperm chalkiness (DEC), chalkiness rate (CR) and chalkiness area (CA) in 12 fragrant japonica varieties and 9 conventional japonica varieties. Furthermore, the endosperm structure and starch granules of rice grains were also observed with scanning electron microscope (SEM). The results indicated that the 21 varieties including the different fragrant japonica and conventional japonica varieties has a linear relationship between the DEC and the CR $(P<0.05)$. Among the fragrant japonica and conventional japonica varieties, the biggest difference is the CR, follow by CA and DEC. In addition, there is a certain correlation between the arrangement of endosperm cells, the distribution of starch granules and the occurrence of chalkiness characters in the different fragrant japonica and conventional japonica varieties. For the same fragrant japonica or conventional japonica variety, there is no significant difference between the starch granules from the transparent parts of chalkiness and non-chalky, while the starch granules of grains between chalky and non-chalky has obvious difference. Our results would provide important references for high quality rice breeding.
\end{abstract}

Keywords: fragrant japonica variety, conventional japonica variety, chalkiness, endosperm, scanning electron microscope

\section{Introduction}

Rice (Oryza sativa L.) is one of the most important food crops in the world, and the staple food for more than half of the world's population (Balindong et al., 2018; Zhao et al., 2013). Along with the sustained development of living standards, people's demand for high quality rice is increasing (Zhou et al., 2015; Fitzgerald et al., 2009; Cheng et al., 2007). However, rice grain quality is a complex quantitative trait which consists of milling, 
appearance, eating, nutritional, cooking and eating qualities (Tian et al., 2009; Peng et al., 2014; Liu et al., 2010). Among these qualities, consumers often focus on appearance quality and cooking and eating quality (Guo et al., 2011; Cuevas et al., 2016). The appearance quality of rice grain, including grain length, grain width, grain thickness and chalkiness or transparency (Qiu et al., 2015), though, grain chalkiness not only affect the rice appearance quality but also influence the milling, cooking and eating qualities (Liu et al., 2010; Guo et al., 2011; Sun et al., 2015; Shen et al., 2007; Yoshioka et al., 2007). Meanwhile, chalkiness characters will reduce head milled rate and thus affect its production in grain, and has a great impact on the price of rice (Cuevas et al., 2016; Sun et al., 2015; Zhao et al., 2013). Therefore, grain chalkiness character is the main determinant of grain qualities and market value of rice.

Chalkiness is one of the main problems in rice production and consumption; there are severe problems of high chalkiness rate and chalkiness degree in many rice varieties. Therefore, reducing the grain chalkiness is an important goal of rice breeders and botanist (Guo et al., 2011; Qiu et al., 2015). Previous studies have indicated that grain chalkiness is a complex character and polygenic controlled quantitative trait loci (QTL) (Peng et al., 2014; Qiu et al., 2015; Liu et al., 2010; Peng et al., 2016), and that there are additive effect and interaction effect between the additive effect and environments (Yoshioka et al., 2007; Li et al., 2014; Tsukaguchi et al., 2008; Peng et al., 2004; Mei et al., 2013; Yamakawa et al., 2008; Wan et al., 2005). At present, more than 140 QTLs controlling grain chalkiness characters have been detected using different type of populations, and these QTLs were distributed on the 12 chromosomes of rice, but, only a few QTLs controlling the trait were obtained for isolation and cloning and function analysis (Peng et al., 2014; Peng et al., 2016; Lin et al., 2016). Some QTLs and genes affecting chalkiness characters were isolated and cloned by natural populations or mutants, i.e. GL7 (Wang et al., 2015), Chalk5 (Li et al., 2014), GW2 (Song et al., 2007), OSPPDKB (Kang et al., 2005), SSIIIa (Fujita et al., 2007; Ryoo et al., 2007), GIF1 (Wang et al., 2010a), OsRab5a (Wang et al., 2010b), flo2 (She et al., 2010). The slow progress in the study of chalkiness partially reflects the complexity of the mechanism underlying grain chalkiness occurrence, which needs intensive studies (Lin et al., 2016). In addition, there is a variety of factors affecting the formation of the grain chalkiness (Yu et al., 2015; Zhao et al., 2015; Zhou et al., 2015), such as starch synthesis, starch granule structure and arrangement, and external environmental factors including high temperature in grain filling period, daily mean temperature, temperature difference of day and night, sunshine duration and cultivation measures.

Compared with non-chalky rice, the chalky grains have the following characteristics, amylose and long branched chain starch contents decreased significantly, short branched chain starch content was significantly increased and head rice percentage and transparency was reduced, consequently, grain eating quality and yield will be debased (Cuevas et al., 2016; Qiu et al., 2015; Patindol et al., 2003). Chalkiness is a white and opaque part in grain, the arrangement of starch granules is loose and inflated, which is result from the influence of development and enrichment of endosperm starch granules and proteoplast at pustulation period (Zhou et al., 2015; Patindol et al., 2003; Li et al., 2014). There are consistent starch granule structure in all rice varieties, therefore, we can preliminary identify the advantages and disadvantages of rice quality using scanning electron microscopy (SEM) (Lisle et al., 2000; Fitzgerald et al., 2009). Most japonica varieties have excellent grain quality, endosperm chalkiness characters are remarkable differences among different rice varieties. Fragrant japonica is a special type of japonica rice and has the higher price due to its fragrance and high nutrition value, which are consider as treasures in rice (Kovach et al., 2009; Daygon et al., 2016; Shao et al., 2013). Previous studies showed that the heritability of grain chalkiness characters is relatively lower in fragrant japonica, and found that chalkiness characters are related to the content and structure of starch (Sun et al., 2015; Zhou et al., 2015; Chakraborty et al., 2016). However, there is still no breakthrough in the progress of mechanism study of grain chalkiness characters formation in japonica varieties especially in fragrant japonica.

In this study, we have mainly investigated the CR, DEC and CA of 12 different fragrant japonica and 9 conventional japonica varieties, and rice endosperm cells and starch granules were observed using SEM. Moreover, the correlation between the formation of chalkiness characters, morphology structure and development of endosperm cells and starch granules were also studied in the 21 rice varieties. Thus, our results will provide useful references for the formation mechanism of grain chalkiness characters in fragrant japonica and conventional japonica varieties, and the grain quality improvement in rice breeding.

\section{Materials and Methods}

\subsection{Materials}

All tested rice varieties are japonica varieties, including 12 fragrant japonica and 9 conventional japonica varieties, and most of them come from China. There are 7 fragrant japonica varieties (Xinxianggeng 1, Xiaobao 
1, Xianggeng 1862, Xiangbao 2, Xianggeng 33, Nongxianggeng, Nongxianggeng 4) and 3 conventional japonica varieties (Zhengdao 02, Zhengdao 18, Xingengnuo 631) from Henan province, 3 fragrant japonica varieties (Xianggeng 805, Wuxianggeng 23, Guanglingxianggeng) and 1 conventional japonica variety (Longgeng 31) from Jiangsu province, 1 fragrant japonica variety (Changxianggeng 101) and 1 conventional japonica variety (Longgeng 31) from Heilongjiang province, 1 conventional japonica variety (Liaoxing 1) from Liaoning province, 1 conventional japonica variety (Xiushui 134) from Zhejiang province, 1 conventional japonica variety (Yilatai 104) from France and 1 conventional japonica variety (Huangjinqing) from Japan. According to the different growth stage of these rice varieties, and to ensure that the 15 japonica varieties which blossom in late August and experience the relatively consistent temperature of grain filling period, these varieties were sowed in batches in a same experiment field of Xinyang City Academy of Agricultural Sciences in 2016 and 2017. Each line was planted with 2 replications in each sowing, with each line containing 12 plants transplanted in a single row with $16.5-\mathrm{cm}$ plant spacing and $26.4-\mathrm{cm}$ row spacing. Field management essentially followed normal agricultural practice. Field irrigation was maintained to avoid drought stress to the late-maturing lines (Peng et al., 2014; Fan et al., 2005), matured rice grains were tested after air drying and room temperature for three months.

\subsection{Determination of Chalkiness Characters}

CR, CA and DEC were measured and analyzed with the National Standard of the People's Republic of China-Good Quality of Rice grains (GB/T17891-1999). In each variety 100 full and complete milled grains were randomly selected, then counted the numbers of grains with chalkiness (repeated 3 times), and the average value was taken as the $\mathrm{CR}$ of each variety; the method of measuring white belly rate and white core rate is similar to the method of CR, i.e. randomly select 100 full and complete milled grains, count the numbers of grains with white belly and white-cored and repeated 3 times, respectively, and the average value is taken as the white belly rate and white core rate; To estimate the percentage of the chalkiness area in whole area of grain, 10 grains with chalkiness were selected randomly in different varieties (repeated 3 times), and the average area is taken as the CA (Peng et al., 2014). DEC is equal to CR multiply by CA [DEC $=$ CR $\times$ CA]. SPSS software was used to analyze the variance of the measured data.

\subsection{Scanning Electron Microscope Observation}

Randomly we selected the grains with chalky and non-chalky in each varieties, for scanning electron microscopy, and milled rice grains were transversely cut at the middle with a knife and coated with gold under HUS-5GB vacuum evaporation equipment. The morphology of starch granules in different part of the endosperm was examined by a scanning electron microscope (Hitachi S-4800). The SEM analysis was based on at least three biological replications of the mounted specimens. All procedures were according to the manufacturer's protocol (Li et al., 2014).

\section{Results}

\subsection{Difference of Chalkiness Characters in Different Fragrant Japonica and Conventional Japonica Varieties}

The results of chalkiness characters in the 12 fragrant japonica varieties and the 9 conventional japonica varieties are shown in Figure 1. Among these japonica varieties, the biggest differences were observed for the CR, follow by CA and DEC. There are higher CR with more than 98\% in Xingengnuo 631, Heixiangdao 193 and Xiangbao 2, while basically no chalkiness characters were observed in Huangjinqing, Nongxianggeng 4 and Longgeng 31 . The CR ranged from $2.9 \%$ to $100 \%$ in the 21 japonica varieties. Variance analysis showed no significant difference of CR among Xinxianggeng 1, Xiangbao 1, Changxianggeng 101 and Wuyungeng 23; also, no significant difference of CR among Xinggengbuo 631, Heixiangdao 193 and Xiangbao 2; likewise, no significant difference of CR among Huangjinqing, Nongxianggeng 4, Longgeng 31 and Xiushui 134; and no significant difference of CR among Nongxianggeng and Yilatai 104. Change trend of DEC was quite similar to the $\mathrm{CR}$ in the 21 japonica varieties, and the DEC ranged between $0.3 \%$ and $100 \%$.

The chalkiness of Changxianggeng 101, Nongxianggeng, Xianggeng 805, Guanglingxianggeng, Zhengdao 02, Liaoxing 1 and Wuyungeng 23 is mainly occurred on the belly of grains, and that is called white belly; the chalkiness characters of Xianggeng 1862, Xiangbao 2, Xianggeng 33 and Yilatai 104 is mainly occurred in center of rice, known as white core. In addition, there are both white belly and white core in Xinxianggeng, Xiangbao 1 and Wuyungeng 23 varieties. However, other varieties' CA was very low and even no-chalky present in grains. The range of CA is from $2.2 \%$ to $100 \%$, and the change trend is different to CR and DEC. The CR of Xinxianggeng 1, Changxianggeng 101 and Xianggeng 805 are all more than $27.0 \%$, but the CA are below $7.0 \%$. Interestingly, the chalkiness characters are almost not observed in Huangjinqing, while the CR is up to $98.4 \%$, CA up to $51.8 \%$, and DEC up to $51 \%$ in Xiangbao 2. Further analysis shows that the linear relationship between 
the CR and DEC was observed in fragrant japonica and conventional japonica varieties $(P<0.05)$, the higher $\mathrm{CR}$ is often accompanied by the higher DEC in the 21 japonica varieties.

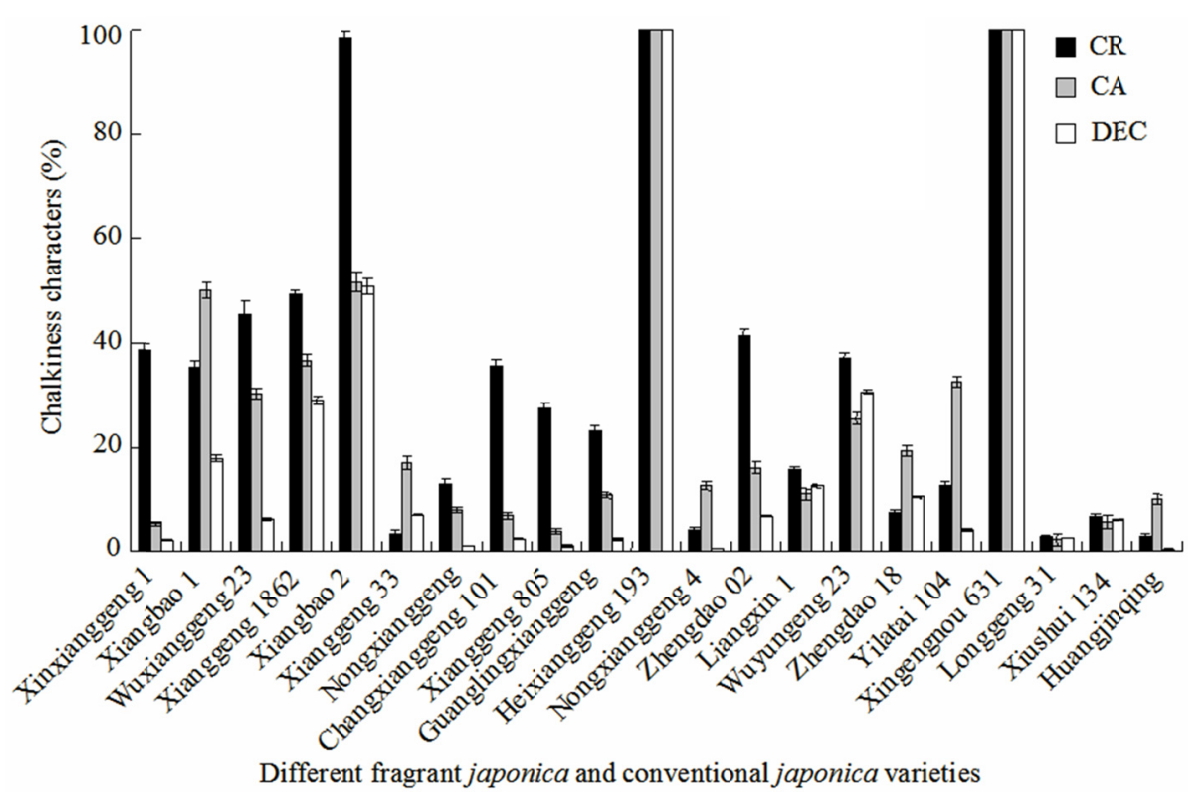

Figure 1. Chalkiness characters of the 21 japonica rice varieties

Note. CR, chalkiness rate; CA, chalkiness area; DEC, degree of endosperm chalkiness.

\subsection{Morphology and Structure of Endosperm Cells in Different Fragrant Japonica and Conventional Japonica Varieties}

From the selected representative milled rice, the arrangement of endosperm cells, cell morphology, cell layer numbers and the distribution of starch granules on the cross section were observed with microstructure and ultrastructure characteristics in the 12 fragrant japonica and the 9 conventional japonica varieties (Figure 2). According to the arrangement of endosperm cells on the cross section, the 21 japonica varieties could be divided into 4 types: the first type is those with radial arrangement in a straight line from the middle of the endosperm cells' cross section to the surrounding, which included the varieties with low CR or even no CR, such as Zhengdao 18, Huangjinqing, Nongxianggeng 4 (Figure 2-1); the second type is those with no radial arrangement from the middle of the endosperm cells' cross section to the surrounding, its included those varieties with mainly white core occurrence, i.e. Yilatai 104, Xianggeng 33, Xiangnuo 1862, Xiangbao 2 (Figure 2-2); the third type is those with apparent radial arrangement in a long line but not straight from the middle of the endosperm cells' cross section to the surrounding, in which including the varieties with mainly white belly occurrence, such as Zhengdao 02, Wuyungeng 23, Nongxiainggeng (Figure 2-3), the last type is those with no evident radial arrangement from the middle of the endosperm cells' cross section to the surrounding, its included those varieties with both white core and white belly, i.e. Xiangbao 1, Xinxianggeng 1, Wuxianggeng 23 (Figure 2-4).

Under the scanning electron microscope, polygonal columnar cells with a more regular arrangement were observed in Zhengdao 18, Huangjinqing and Nongxianggeng 4, but irregular cells was not obvious and even could not be observed in these varieties (Figure 2-1c). Columnar cells with multilayer and polygonization, in mainly occurred grains with white core, were observed in Yilatai 104, Xianggeng 33, Xiangnuo 1862, Xiangbao 2, while there were many oval and irregular cells around the columnar cells (Figure 2-2c). Irregular cell groups and columnar cells with multilayer and polygonization were also observed in Zhengdao 02, Wuyungeng 23 and Nongxiainggeng, which have grains occurred mainly with white belly (Figure 2-3c). Moreover, oval cell groups and columnar cells with polygonization around the center were observed in Xiangbao 1, Xinxianggeng 1, Wuxianggeng 23, both white core and white belly grains existent in these varieties (Figure 2-4c). 

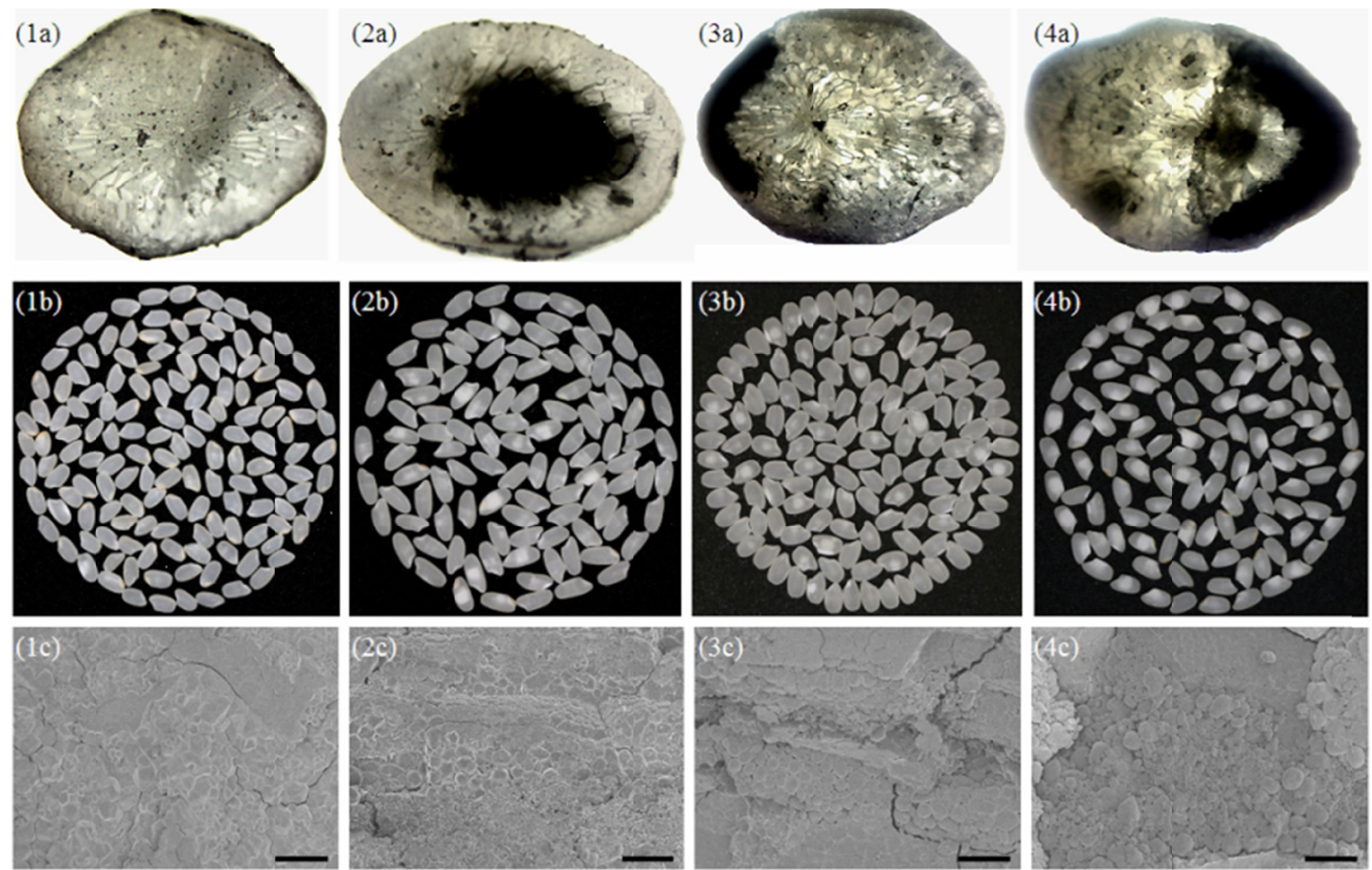

Figure 2. Low magnification view of the chalkiness characters of the tested rice varieties

Note. Low magnification view of transversely fractured midregion ( $a, c)$ and milled rice (b); 1 4, Huangjinqing, Yilatai 104, Wuyungeng 23, Xiangbao 1; Bar $=40 \mu \mathrm{m}$.

The distribution of starch granules in rice grains was also observed by SEM, we can see that starch granules are non-uniform evidently in the chalkiness grains of the fragrant japonica varieties and conventional japonica varieties, and starch granules mainly concentrated on the center part and dorsoventral direction (Figure 2). In addition, the density of starch granules was different among the diverse japonica varieties. For the core white based rice varieties, the starch granules were mainly distributed in the central part of the grain, while the starch granules were distributed in the belly of the grain is white belly based rice varieties. Moreover, the varieties in which the starch granules were distributed on the belly and central part of grains were those japonica varieties with both white core and white belly. However, starch granules were distributed evenly and apparently in the cross-section and the density of starch granules is large in fragrant japonica and conventional japonica varieties with very low CR or even no chalkiness (Figure 2). These results indicated that there are certain correlation between arrangement of endosperm cells, distribution of starch granules and occurrence of grain chalkiness characters in fragrant japonica and conventional japonica varieties.

\subsection{Starch Granules of Chalkiness Grain and Non-chalky Grain in Fragrant Japonica and Conventional Japonica Varieties}

Chalkiness grain and non-chalky grain were also observed by SEM in the 12 fragrant japonica and 9 conventional japonica varieties. Our results showed that starch granules in the grain chalkiness region is a sphere or ellipsoid, the arris is not obvious, starch granules size is uneven and the arrangement is irregular, though, many free single starch granules arranged loosely were also observed, and at the same time the gap of the starch granules were big (Figures 3-a and 3-d). Therefore, the development of endosperm cells is not good in the chalkiness region, and the accumulation of starch granules is irregular.

There was no significant difference of the starch granules between the transparent parts of the chalkiness grain and the non-chalky grain. Most of the starch granules in the transparent parts of the non-chalky grain were in the rules of the diamond or other polygons with polyhedral arris; identical size and dense arrangement with the gaps were small or even none existent (Figures 3-b, 3-c, 3-e, and 3-f). The results indicated the close correlation between the shape and arrangement of starch granules with occurrence of grain chalkiness characters in fragrant 
japonica and conventional japonica varieties. Thus, the starch granules developed well in the low chalkiness or non-chalky grain, conversely, the starch granules developed poorly.
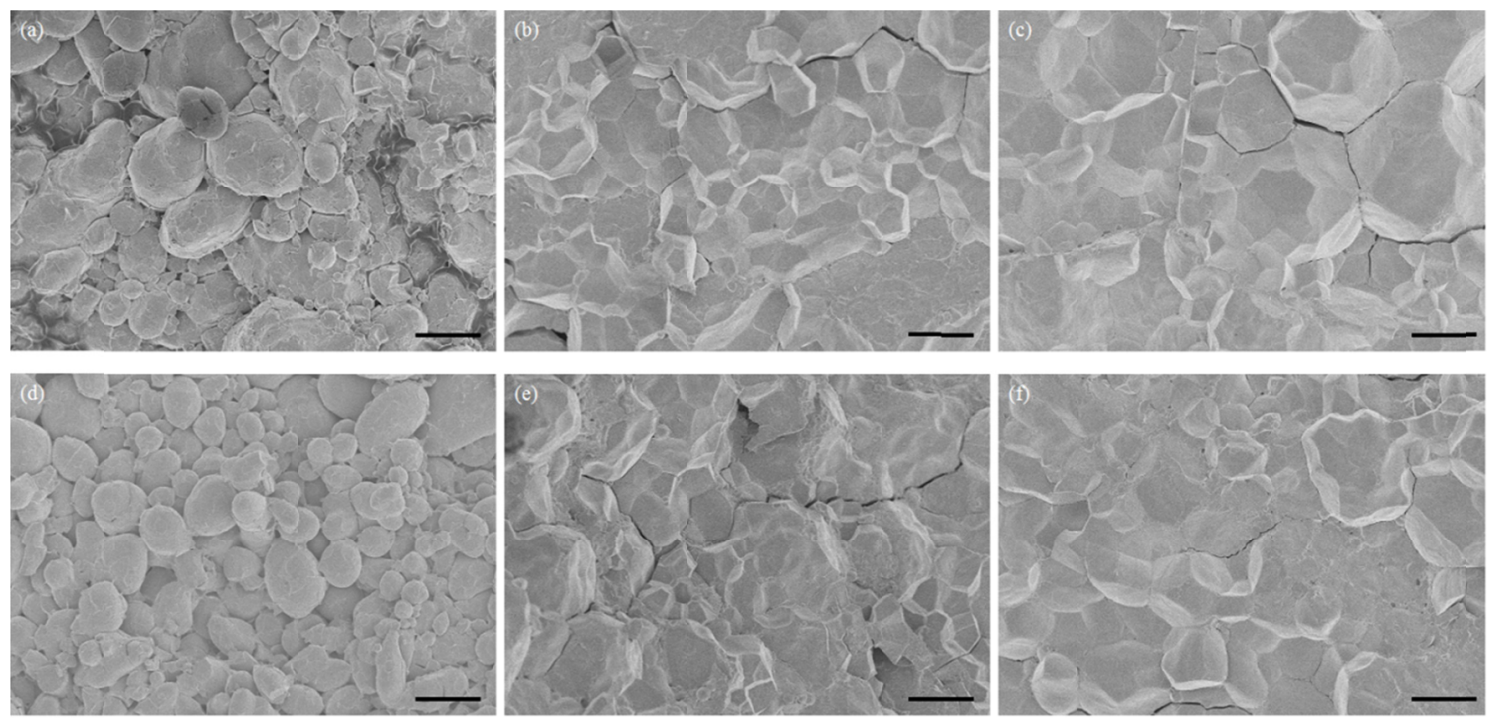

Figure 3. Scanning electron microscopic observation on starch granules in endosperm cells of the chalky and non-chalky grains

Note. a, d: the starch grains in the chalky rice; b, e: the starch grains in the transparent parts of rice; c, f: the starch grains in the non-chalky rice; $\mathrm{Bar}=8 \mu \mathrm{m}$.

\subsection{Morphology of Starch Granules in Different Parts of the Grains From Fragrant Japonica and Conventional Japonica Varieties}

According to the different parts of grain chalkiness in rice endosperm, the chalkiness can be divided into white belly, white core and white back (Qiu et al., 2015; Peng et al., 2014). Under the SEM, the belly, core and back of chalkiness rice were observed in the 12 fragrant japonica and the 9 conventional japonica varieties, respectively. The results showed that the gap was big in the center of endosperm, starch granules arranged loosely, and the size of starch granules was irregular and the development was poor in these varieties, i.e. Yilatai 104, Xianggeng 33, Xiangnuo 1862 and Xiangbao 2, though the starch granules of belly and back in grain endosperm present a typical diamond shape and the arris is sharp and arrange closely, simultaneously present mostly in the composite starch granules and develop well (Figure 4-1). The starch granules in belly were in the big gap, with identical size, loose arrangement and poor development, but those in the center and back of grain endosperm parts were in the typical diamond shape and the arris is sharp and arrange closely; However, in general, these varieties of Zhengdao 02, Wuyungeng 23, Nongxianggeng develop well (Figure 4-2). Moreover, there are many free starch granules with identical size and poor development in the center and belly of grain endosperm cells in Xiangbao 1 , Xingxianggeng 1 and Wuxianggeng 23, while the starch granules of grain back develop well (Figure 4-3). For the varieties of Zhengdao 18, Huangjinqing and Nongxianggeng 4, it commonly showed typical diamond with dense arrangement and many composite starch granules in the center, belly and back of endosperm cells, and all of the starch granules developed well (Figure 4-4). Through the morpholog observation of starch granules of different parts on chalkiness grain, no special morphology of starch granules was observed among the 12 fragrant japonica and the 9 conventional japonica varieties. Consequently, there is a direct relationship between the occurrence of grain chalkiness and development of starch granules in fragrant japonica and conventional japonica varieties.

\section{Discussion}

\subsection{Grain Chalkiness Characters and Distribution of Starch Granules in Fragrant Japonica and Conventional Japonica Varieties}

Grain chalkiness affects both the appearance and processimg qualities and even the cooking and eating oncs, thus it is a very important quality trait in rice (Peng et al., 2016; Peng et al., 2017; Qiu et al., 2015). The scanning 
electron microscope (SEM) techniques is usually used to observe directly the morphological structure of starch granules in grains, and facilitate the preliminarily identification of the quality of grains ( $\mathrm{Li}$ et al., 2014; Lisle et al., 2000). In the present study, through observation by SEM and analysis of 12 fragrant japonica and 9 conventional japonica varieties, we found that the starch granules in the cross section of the grain endosperm was significantly uneven in the varieties with the higher CR and DEC (i.e. Xingengnuo 631, Heixianggeng 193 and Xiangbao 2), while the starch granules in the center, belly and back of the grain endosperm was all more even in the varieties with the lower CR and DEC (i.e. Huangjinqing, Nongxianggeng 4 and Longgeng 31). Further more, the comprehensive analysis of the chalkiness characters and the cells' morphological structure of the grain cross section, showed that if the distribution of starch granules is relatively uniform on the cross section, the CR and DEC will be lower, thus it will not be easy to produce chalkiness in grains; if the distribution of starch granules is uneven and arrange loosely, starch granules' gap is big and dysplasia on the cross section, so the CR and DEC will be higher and it is more likely to easily produce the chalkiness. Therefore, the distribution of starch granules in the cross section of grain endosperm has an important role influencing the occurrence of chalkiness characters in fragrant japonica and conventional japonica varieties.
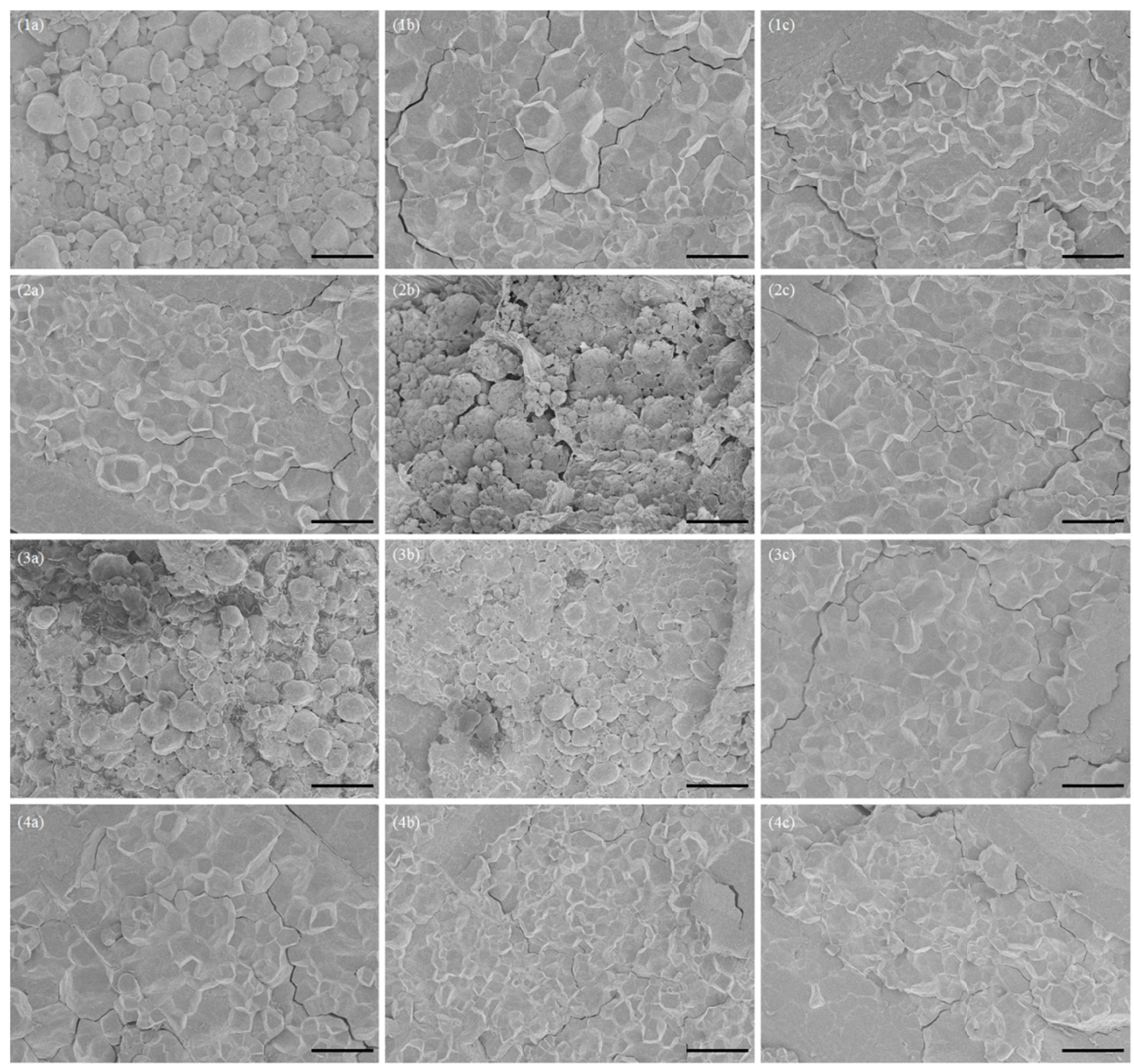

Figure 4. Scanning electron microscopic observation on starch granules in different part of the japonica rice varieties

Note. 1 4, Yilatai 104, Wuyungeng 23, Xiangbao 1, Huangjinqing; a, starch grains in the center of the endosperm; $b$, starch grains in the endosperm of the belly; $c$, starch grains on the back of the endosperm; Bar = $20 \mu \mathrm{m}$. 


\subsection{Grain Chalkiness and Development of Starch Granules in Fragrant Japonica and Conventional Japonica Varieties}

In this study, the observation of chalkiness characters in the 12 fragrant japonica varieties and the 9 conventional japonica varieties, Showed that the morphological structure and arrangement of starch granules in grain endosperm cells were different in the different parts of the same japonica varieties. Compared with the starch granules from the grains of transparent parts or without grain chalkiness varieties, the starch granules were mainly in the free state of existence and cannot further developed into certain form of starch granules complex in fragrant japonica and conventional japonica varieties. So, if the individual starch granules can be closely ordered into some form of starch granules complex, the grain chalkiness characters would not occurre at this site. Though, if the spherical or ellipsoidal starch granules is arranged irregularly in the grain chalkiness regions, the deposition showed disordered state and the deposition of starch granules are reflected in the appearance of the grains, which means that the grain endosperm has chalkiness characters and its performance is not the transparent state. In addition, previous studies have shown that the development in the back of endosperm cells is relatively better than that in the center and belly of grain endosperm, which showed so little or no white back in grains (Li et al., 2014; Sing et al., 2003; Kim et al., 2004). We also found no white back in grains, and starch granules of back are in the rules of the diamond or other polygons with polyhedral arris, dense arrangement, small and even no gap between starch granules and the starch granules of back developed well in grains. When testing endosperm of 12 fragrant japonica and 9 conventional japonica varieties in this study, the reason could be that nutrients are first transported to the back of the japonica rice seeds, and then to the central part of the endosperm and the abdomen, this makes the development of back of the grain endosperm relatively good. Therefore, we may say that there is close relationship between grain chalkiness characters of endosperm and development of starch granules in fragrant japonica and conventional japonica varieties.

\subsection{Reasons for the Formation of Grain Chalkiness}

Previous studies have shown that grain chalkiness is a complex quantitative trait, which is controlled by multiple genes and readily influenced by environmental factors (Qiu et al., 2015; Guo et al., 2011; Li et al., 2014; Tsukaguchi et al., 2008; Peng et al., 2014; Peng et al., 2016). In this study, among the 12 fragrant japonica and the 9 conventional japonica varieties, there were obvious differences in chalkiness characters in grain endosperm, and if we combined it with our preliminary findings, we can concurre that chalkiness characters of grain endosperm are mainly controlled by genes. However, it is also known that the occurrence of grain chalkiness is a very complex process, in which the relationship between the "source", "sink" and "flow" may be involved in the formation of endosperm chalkiness (Lin et al., 2016; Kim et al., 2004). Thus, a change or uncoordinated in any of these three relations are likely to cause or increase the opportunity of the formation of endosperm chalkiness characters. In regard of what preceded, we cool consider that endosperm cells and the structure of starch granules on the cross section determine grain chalkiness characters, and endosperm structure is influenced by the development of rice endosperm cells, which are all controlled by genetic factors. Therfore, the happening of grain chalkiness in fragrant japonica and conventional japonica varieties is intimately related to the distribution and development of starch granules in certain region of grain endosperm, and it could be very critical for the occurrence, development, later deposition patterns and distribution ways of starch granules in rice endosperm. However, little is known about the starch granules of early occurrence, development and later deposition pattern in fragrant japonica and conventional japonica varieties. Indeed, many questions still unanswered up to now, such us, how many genes might be involved in this important regulation process? The interaction of these genes? How is played the key role for starch granules in the process of chalkiness formation in fragrant japonica and conventional japonica varieties? The solution of these crucial issues) will provide important information for further clarifying the mechanism of the occurrence of grain chalkiness characters in rice.

Of all what preceed, the present study found that both the arrangement of endosperm cells and distribution of starch granules are associated with the occurrence of grain chalkiness in fragrant japonica and conventional japonica varieties. Therefore, we can easily say that our results could help to pave a new way for the improvement of grain chalkiness and grain quality in japonica varieties and, potentially, other staple crops.

\section{Acknowledgements}

This work was financially supported by National Natural Science Foundation of China (U1604110, U1404319, 31600992), Key Project of Science and Technology in Henan Province (182102110442, 152102110100, 152102110036), Students Innovation and Training Program of Institution of Higher Learning in Henan Province (201710477018Y); Nanhu Scholars Program for Young Scholars of XYNU (2016054), Students Innovation and Training Program of XYNU (201710477032); Scientific Research Innovation Project for Postgraduate of 
XYNU(2017KYJJ44); Major Science and Technology Project in Henan Province (121100110200) and Institute for Conservation and Utilization of Agro-bioresources in Dabie Mountains.

\section{References}

Balindong, J. L., Ward, R. M., Liu, L., Rose, T. J., Pallas, L. A., Ovenden, B. W., .. Waters, D. L. E. (2018). Rice grain protein composition influences instrumental measures of rice cooking and eating quality. Journal of Cereal Science, 79, 35-42. https://doi.org/10.1016/j.jcs.2017.09.008

Chakraborty, R., Suvra, T., Quamruzzaman, M., Bandopaddhya, N., \& Rasul, M. G. (2016). Performance of legendary local fragrant rice in bangladesh. Journal of Agriculture and Ecology Research International, 6(4), 1-7. https://doi.org/10.9734/JAERI/2016/23009

Cheng, S. H., Zhuang, J., Fan, Y. J., \& Gao, L. (2007). Progress in research and development on hybrid rice: A super-domesticate in China. Annals of Botany, 100(5), 959-966. https://doi.org/10.1093/aob/mcm121

Cuevas, R. P., Pede, V. O., McKinley, J., Velarde, O., \& Demon, M. (2016). Rice grain quality and consumer preferences: A case study of two rural towns in the Philippines. PLOS ONE, 11(3), e0150345. https://doi.org/ 10.1371/journal.pone.0150345

Daygon, V. D., Prakash, S., Calingacion, M., Riedel, A., Ovenden, B., Snell, P., ... Fitzgerald, M. (2016). Understanding the jasmine, phenotype of rice through metabolite profiling and sensory evaluation. Metabolomics, 12(4), 1-15. https://doi.org/10.1007/s11306-016-0989-6

Fan, C. C., Yu, X. Q., Xing, Y. Z., Xu, G. G., Li, X. H., \& Zhang, Q. F. (2005). The main effects, epistatic effects and environmental interactions of QTLs on the cooking and eating quality of rice in a doubled-haploid line population. Theoretical and Applied Genetics, 110(8), 1445-1452. https://doi.org/10.1007/s00122-0051975-y

Fitzgerald, M. A., McCouch, S. R., \& Hall, R. D. (2009). Not just a grain of rice: The quest for quality. Trends in Plant Science, 14(3), 133-139. https://doi.org/10.1016/j.tplants.2008.12.004

Fujita, N., Yoshida, M., Kondo, T., Saito, K., Utsumi, Y., Tokunaga, T., ... Nakamura, Y. (2007). Characterization of SSIIIa-deficient mutants of rice: The function of SSIII and pleiotropic effects by SSIIIa deficiency in the rice endosperm. Plant Physiology, 144(4), 2009-2023. https://doi.org/10.1104/pp.107.102533

Guo, T., Liu, X. L., Wan, X. Y., Weng, J. F., Liu, S. J., Liu, X., ... Wan, J. M. (2011). Identification of a stable quantitative trait locus for percentage grains with white chalkiness in rice. Journal of Integrative Plant Biology, 53(8), 598-607. https://doi.org/10.1111/j.1744-7909.2011.01041.x

Kang, H. G., Park, S., Matsuoka, M., \& An, G. (2005). White-core endosperm floury endosperm-4 in rice is generated by knockout mutations in the C-type pyruvate orthophosphate dikinase gene (OSPPDKB). Plant Journal, 42(6), 901-911. https://doi.org/10.1111/j.1365-313X.2005.02423.x

Kim, K. S., Kang, H. J., Hwang, I. K., Hwang, H. G., Kim, T. Y., \& Choi, H. C. (2004). Comparative ultrastructure of ilpumbyeo, a high-quality Japonica rice, and its mutant suweon 464: Scanning and transmission electron microscopy studies. Journal of Agricultural Food Chemistry, 52(12), 3876-3883. https://doi.org/10.1021/jf049767r

Kovach, M. J., Calingacion, M. N., Fitzgerald, M. A., \& McCouch, S. R. (2009). The origin and evolution of fragrance in rice (Oryza sativa L.). Proceedings of the National Academy of Sciences of the United States of America, 106(34), 14444-14449. https://doi.org/10.1073/pnas.0904077106

Li, Y. B., Fan, C. C., Xing, Y. Z., Yun, P., Luo, L. J., Yan, B., ... He, Y. Q. (2014). Chalk5 encodes a vacuolar $\mathrm{H}^{+}$-translocating pyrophosphatase influencing grain chalkiness in rice. Nature Genetics, 46(4), 389-404. https://doi.org/10.1038/ng.2923

Lin, Z. M., Zheng, D. Y., Zhang, X. C., Wang, Z. X., Lei, J. C., Liu, Z. H., ... Ding, Y. F. (2016). Chalky part differs in chemical composition from translucent part of japonica rice grains as revealed by a notched-belly mutant with white-belly. Journal of the Science of Food and Agriculture, 96(11), 3397-3343. https://doi.org/ $10.1002 /$ jsfa. 7793

Lisle, A. J, Matin, M., \& Fitzgerald, M. A. (2000). Chalky and translucent rice grains differ in starch composition an structure and cooking properties. Cereal Chemistry, 77(5), 627-632. https://doi.org/10.1094/ CCHEM.2000.77.5.627 
Liu, X. L., Guo, T., Wan, X. Y., Wang, H. Y., Zhu, M. Z., Li, A. L., .. Wan, J. M. (2010). Transcriptome analysis of grain-filling caryopses reveals involvement of multiple regulatory pathways in chalky grain formation in rice. BMC Genomics, 11(1), 730. https://doi.org/10.1186/1471-2164-11-730

Mei, D. Y., Zhu, Y. J., Yu, Y. H., Fan, Y. F., Huang, D. R., \& Zhuang, J. Y. (2013). Quantitative trait loci for grain chalkiness and endosperm transparency detected in three recombinant inbred line populations of indica rice. Journal of Integrative Agriculture, 12(1), 1-11. https://doi.org/10.1016/S2095-3119(13)60199-9

Patindol, J., \& Wang, Y. J. (2003). Fine structures and physicochemical properties of starches from chalky and translucent rice kernels. Journal of Agricultural and Food Chemistry, 51(9), 2777-2784. https://doi.org/ $10.1021 / \mathrm{jf026101 \textrm {t }}$

Peng, B., Sun, Y. F., Kong, D. Y., Pang, R. H., Song, X. H., Li, H. L., ... Yuan, H. Y. (2017). Chalkiness charactersand scanning electron microscope observaion of rice grain endosperm of japonica varietiesin Sunther Henan. Asian Agricultural Research, 9(3), 80-85.

Peng, B., Sun, Y. F., Li, Q. R., Li, D., Pang, R. H., Zhou, Q. Y., ... Song, S. Z. (2016). Progress in genetic research on rice chalkiness. Journal Xinyang Normal University, 29(2), 304-312. https://doi.org/10.3969/ j.issn.1003-0972.2016.02.035

Peng, B., Wang, L. Q., Fan, C. C., Jiang, G. H., Luo, L. J., Li, Y. B., \& He, Y. Q. (2014). Comparative mapping of chalkiness components in rice using five populations across two environments. BMC Genetics, 15, 49. https://doi.org/10.1186/1471-2156-15-49

Peng, S. B., Huang, J. L., Sheehy, J. E., Laza, R. C., Visperas, R. M., Zhong, X. H., .. Cassman, K. G. (2004). Rice yields decline with higher night temperature from global warming. Proceedings of the National Academy of Sciences of the United States of America, 101(27), 9971-9975. https://doi.org/10.1073/ pnas. 0403720101

Qiu, X. J., Pang, Y. L., Yuan, Z. H., Xing, D. Y., Xu, J. L., Dingkuhn, M., ... Ye, G. Y. (2015). Genome-wide association study of grain appearance and milling quality in a worldwide collection of indica rice germplasm. PLoS ONE, 10(12), e0145577. https://doi.org/10.1371/journal.pone.0145577

Ryoo, N., Yu, C., Park, C. S., Baik, M. Y., Park, I. M., Cho, M. H., ... Jeon, J. S. (2007). Knockout of a starch synthase gene OsSSIIIa/Flo5 causes white-core floury endosperm in rice (Oryza sativa L.). Plant Cell Reports, 26(7), 1083-1095. https://doi.org/10.1007/s00299-007-0309-8

Shao, G. N., Tang, S. Q., Chen, M. L., Wei, X. J., He, J. W., Luo, J., ... Hu, P. S. (2013). Haplotype variation at Badh2, the gene determining fragrance in rice. Genomics, 101(2), 157-162. https://doi.org/10.1016/ j.ygeno.2012.11.010

She, K. C., Kusano, H., Koizumi, K., Yamakawa, H., Hakata, M., Imamura, T., ... Shimada, H. (2010). A novel factor floury endosperm 2 is involved in regulation of rice grain size and starch quality. Plant Cell, 22(10), 3280-3294. https://doi.org/10.1105/tpc. 109.070821

Shen, X. P., Shen, X. Y., Gu, L., Gong, L. P., \& Zhang, H. C. (2007). Effect of seeding time on chalkiness of liangyoupeijiu in Jiangsu rice growing areas at different latitudes. Chinese Journal of Rice Science, 21(6), 677-680. https://doi.org/10.16819/j.1001-7216.2007.06.020

Singh, N., Sodhi, N. S., Kaur, M., \& Saxena, S. K. (2003). Phyicochemical, morphological, thermal, cooking and textural properties of chalky and translucent rice kernels. Food Chemistry, 82(3), 433-439. https://doi.org/ 10.1016/S0308-8146(03)00007-4

Song, X. J., Huang, W., Shi, M., Zhu, M. Z., \& Lin, H. X. (2007). A QTL for rice grain width and weight encodes a previously unknown RING-type E3 ubiquitin ligase. Nature Genetics, 39(5), 623-630. https://doi.org/10.1038/ng2014

Sun, W. Q., Zhou, Q. L., Yao, Y., Qiu, X. J., Xie, K., \& Yu, S. B. (2015). Identification of genomic regions and the isoamylase gene for reduced grain chalkiness in rice. PLOS ONE, 10(3), e0122013. https://doi.org/ 10.1371/journal.pone.0122013

Tian, Z. X., Qian, Q., Liu, Q. Q., Yan, M. X., Liu, X. F., Yan, C. J., ... Li, J. Y. (2009). Allelic diversities in rice starch biosynthesis lead to a diverse array of rice eating and cooking qualities. Proceedings of the National Academy of Sciences of the United States of America, 106(51), 21760-21765. https:/doi.org/10.1073/ pnas.0912396106 
Tsukaguchi, T., \& Lida, Y. (2008). Effects of assimilate supply and high temperature during grain-filling period on the occurrence of various types of chalky kernel in rice plants (Oryza sativa L.). Plant Production Science, 11(2), 203-210. https://doi.org/10.1626/pps.11.203

Wan, X. Y., Wan, J. M., Weng, J., Jiang, L., Bi, J. C., Wang, C. M., \& Zhai, H. Q. (2005). Stability of QTLs for rice grain dimension and endosperm chalkiness characteristics across eight environments. Theoretical and Applied Genetics, 110(7), 1334-1346. https://doi.org/10.1007/s00122-005-1976-x

Wang, E. T., Xu, X., Zhang, L., Zhang, H., Lin, L., Wang, Q., ... He, Z. H. (2010a). Duplication and independent selection of cell-wall invertase genes GIF1 and OSCIN1 during rice evolution and domestication. BMC Evolutionary Biology, 10(1), 1-13. https://doi.org/10.1186/1471-2148-10-108

Wang, Y. H., Ren, Y. L., Liu, X., Jiang, L., Chen, L. M., Han, X. H., ... Wan, J. M. (2010b). OsRab5a regulates endomembrane organization and storage protein trafficking in rice endosperm cells. Plant Journal, 64(5), 812-824. https://doi.org/10.1111/j.1365-313X.2010.04370.x

Wang, Y. X., Xiong, G. S., Hu, J., Jiang, L., Yu, H., Xu, J., Fang, Y. X., ... Qian, Q. (2015). Copy number variation at the GL7 locus contributes to grain size diversity in rice. Nature Genetics, 47(8), 944-948. https://doi.org/10.1038/ng.3346

Yamakawa, H., Ebitani, T., \& Terao, T. (2008). Comparison between locations of QTLs for grain chalkiness and genes responsive to high temperature during grain filling on the rice chromosome map. Breeding Science, 58(3), 337-343. https://doi.org/10.1270/jsbbs.58.337

Yoshioka, Y., Iwata, H., Tabata, M., Ninomiya, S., \& Ohsawa, R. (2007). Chalkiness in rice: Potential for evaluation with image analysis. Crop Science, 47(5), 2113-2120. https://doi.org/10.2135/cropsci2006. $10.0631 \mathrm{sc}$

Yu, L., Liu, Y. H., Tong, J. H., Ding, J. H., Wang, R. Z., Peng, C. L., \& Xiao, L. T. (2015). Reduced grain chalkiness and its possible physiological mechanism in transgenic rice overexpressing Lgalld. Crop Journal, 3(2), 125-134. https://doi.org/10.1016/j.cj.2014.12.001

Zhao J. L., Zhao, C. J., Yang, H., Zhang, D. Y., Dong, Y. Y., \& Yuan, L. (2013). Identification and characterization of spectral response properties of rice canopy infested by leaf folder. International Journal of Agriculture Biology, 15(4), 694-700. https://doi.org/12-1105/2013/15-4-694-700

Zhao, X. Q., \& Fitzgerald, M. (2013). Climate change: Implications for the yield of edible rice. PLoS One, 8(6), e66218. https://doi.org/10.1371/journal.pone.0066218

Zhao, X. Q., Daygon, V. D., McNally, K. L., Hamilton, R. S., Xie, F. M., Reinke, R. F., \& Fitzgerald, M. A. (2015). Identification of stable QTLs causing chalk in rice grains in nine environments. Theoretical and Applied Genetics, 129(1), 1-13. https://doi.org/10.1007/s00122-015-2616-8

Zhou, L. J., Liang, S. S., Ponce, K., Marundon, S., Ye, G. Y., \& Zhao, X. Q. (2015). Factors affecting head rice yield and chalkiness in indica rice. Field Crops Research, 172, 1-10. https://doi.org/10.1016/j.fcr.2014. 12.004

\section{Copyrights}

Copyright for this article is retained by the author(s), with first publication rights granted to the journal.

This is an open-access article distributed under the terms and conditions of the Creative Commons Attribution license (http://creativecommons.org/licenses/by/4.0/). 